03,13

\title{
Эволюция ансамбля микропор в структуре SiC/Si в процессе роста методом замещения атомов
}

\author{
(C) А.В. Редьков ${ }^{1,2}$, А.С. Гращенко ${ }^{1,2}$, С.А. Кукушкин ${ }^{1,2,3}$, А.В. Осипов ${ }^{1,2}$, К.П. Котляр $p^{4,5}$, \\ А.И. Лихачев ${ }^{4,5}$, А.В. Нащекин ${ }^{4,5}$, И.П. Сошников ${ }^{4,5}$ \\ ${ }^{1}$ Институт проблем машиноведения РАН, \\ Санкт-Петербург, Россия \\ ${ }^{2}$ Санкт-Петербургский национальный исследовательский университет информационных технологий, механики и оптики, \\ Санкт-Петербург, Россия \\ ${ }^{3}$ Санкт-Петербургский политехнический университет им. Петра Великого, \\ Санкт-Петербург, Россия \\ ${ }^{4}$ Санкт-Петербургский академический университет РАН, \\ Санкт-Петербург, Россия \\ ${ }^{5}$ Физико-технический институт им. А.Ф. Иофрфе РАН, \\ Санкт-Петербург, Россия \\ E-mail: avredkov@gmail.com
}

(Получена 22 октября 2018 г.)

Исследована временна́я эволюция ансамбля микропор, формирующихся в приповерхностной области кремния в процессе роста тонких пленок карбида кремния методом замещения атомов. Образцы $\mathrm{SiC} / \mathrm{Si}$ исследованы методом сканирующей электронной микроскопии, эллипсометрии и конфокальной рамановской микроскопии. Показаны характерные этапы формирования пористого слоя: зарождение одиночных пор, их рост с образованием дендритоподобных структур и последующее срастание в сплошной слой. Продемонстрировано, что толщина пористого слоя на начальных этапах роста пропорциональна корню кубическому из времени. Обсуждаются возможные механизмы формирования пор и предложена теоретическая модель для описания зависимости средней толщины пористого слоя от времени, которая имеет хорошее качественное совпадение с экспериментальными результатами.

Работа поддержана Российским научным фондом (грант \# 14-12-01102).

DOI: 10.21883/FTT.2019.03.47232.265

\section{1. Введение}

Одним из актуальных направлений развития электроники в последние десятилетия являются широкозонные полупроводники группы III-V, в частности нитриды алюминия и галлия $(\mathrm{AlN}, \mathrm{GaN})[1,2]$, на основе которых уже создаются новые поколения высокопроизводительных и энергоэффективных электронных устройств $[3,4]$. В связи с этим большое количество исследований посвящено именно им. Поскольку собственные подложки III-нитридов в настоящий момент малодоступны, продолжается поиск подложек иных материалов, обеспечивающих бездефектную гетероэпитаксию этих полупроводников. В промышленности в качестве подложек чаще всего используются сапфир, кремний и карбид кремния, как с дополнительными буферными слоями, так и без [5-7]. Одним из перспективных, на наш взгляд, кандидатов на роль подложки для промышленного производства III-нитридов являются подложки кремния $(\mathrm{Si})$ с буферным слоем карбида кремния (SiC), которые объединяют преимущества как $\mathrm{SiC}$, так и $\mathrm{Si}[8]$. В серии работ, обобщенных в обзорах $[8,9]$, был теоретически предсказан и экспериментально подтвержден простой и доступный метод формирования подобных тонких пленок $\mathrm{SiC} / \mathrm{Si}$ путем частичного замещения атомов кремния на углерод непосредственно в приповерхностной области кремния, которое происходит в результате топохимической реакции [9]

$$
2 \mathrm{Si}_{\text {solid }}+\mathrm{CO}_{\text {gas }} \rightarrow \mathrm{SiC}_{\text {solid }}+\mathrm{SiO}_{\text {gas }}
$$

Характерной особенностью этого процесса является то, что ему сопутствует образование большого количества пор под поверхностью новообразованного слоя $\mathrm{SiC}$ [9]. Подобный пористый слой может сыграть позитивную роль при дальнейшем росте последующих тонких слоев III-нитридов [10,11], поскольку позволяет сократить механическое влияние кремния вследствие уменьшенной площади контакта пленки и подложки [9]. Так, в работах $[12,13]$ было изучено влияние искусственно сформированных пор в структуре $\mathrm{SiC} / \mathrm{Si}$ на рост нитридов алюминия и галлия и было показано, что наличие подобных пор может привести к росту последующих пленок более высокого качества.

В связи с этим, представляется целесообразным исследование процессов и закономерностей, приводящих к порообразованию, а также поиск методов для оценки конечного распределения пор под поверхностью $\mathrm{SiC}$ в зависимости от условий роста. Настоящая работа направлена на изучение механизмов формирования наблюдаемых пор и временно́й эволюции их среднего размера. В первой части работы приведены экспериментальные 

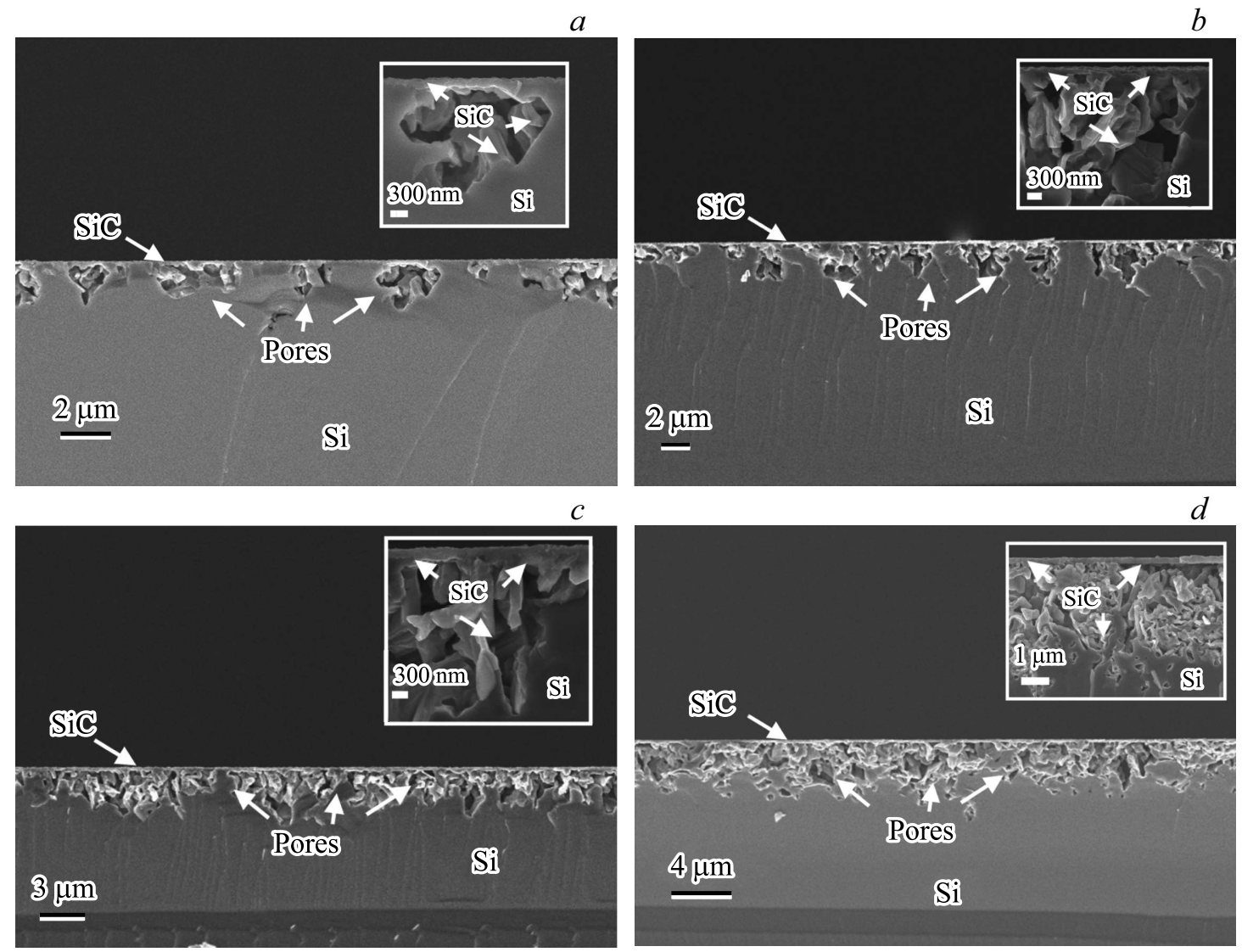

Рис. 1. СЭМ-изображение сколов пористой структуры $\mathrm{SiC} / \mathrm{Si}$, образовавшейся после отжига кремния в $\mathrm{CO}$ в течение: 1 min $(a)$, $3.5 \min (b), 12 \min (c), 40 \min (d)$. Врезки демонстрируют увеличенное изображение пор.

результаты по исследованию формирующихся пористых слоев. Вторая часть посвящена обсуждению механизмов формирования пористого слоя и теоретической модели, позволяющей оценить зависимость толщины пористого слоя от условий отжига кремния в атмосфере СО по реакции (1).

\section{2. Эксперимент}

Для изготовления подложек $\mathrm{SiC} / \mathrm{Si}$ были выбраны пластины кремния ориентации (100), которые отжигались в атмосфере газа СО в присутствии силана $\left(\mathrm{SiH}_{4}\right)$ по технологическому процессу и в реакторе, описание которых доступно в обзорах $[8,9]$. Серия из четырех образцов была изготовлена при одних и тех же условиях, представленных в таблице, за исключением

Условия синтеза тонких пленок $\mathrm{SiC} / \mathrm{Si}$

\begin{tabular}{l|c}
\hline Температура, ${ }^{\circ} \mathrm{C}$ & 1290 \\
\hline Общее давление в реакторе $\left(\mathrm{CO}+\mathrm{SiH}_{4}\right)$, Torr & 0.5 \\
\hline Поток газовой смеси $\left(\mathrm{CO}+\mathrm{SiH}_{4}\right), \mathrm{ml} / \mathrm{min}$ & 13 \\
\hline Доля силана в газовой смеси, \% & 5.5
\end{tabular}

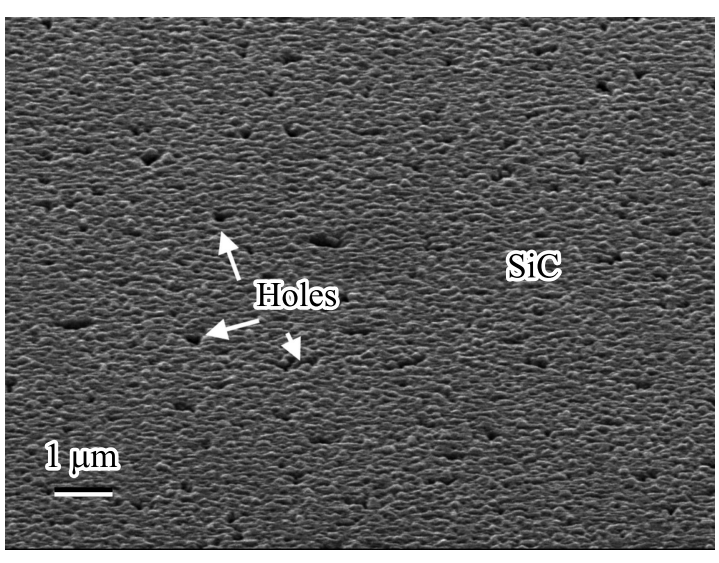

Рис. 2. СЭМ-изображение отверстий на поверхности образца $\mathrm{Si}$, отожженного в атмосфере $\mathrm{CO}$ в течение $1 \mathrm{~min}$.

длительности отжига, которая составила для первого образца $1 \mathrm{~min}$, а для последующих, соответственно, 3.5, 12 , и $40 \mathrm{~min}$.

Образцы были исследованы методом сканирующей электронной микроскопии (СЭМ), оптической микроскопии, эллипсометрии, а также конфокальной рамановской микроскопии. СЭМ-изображения сколов образцов 


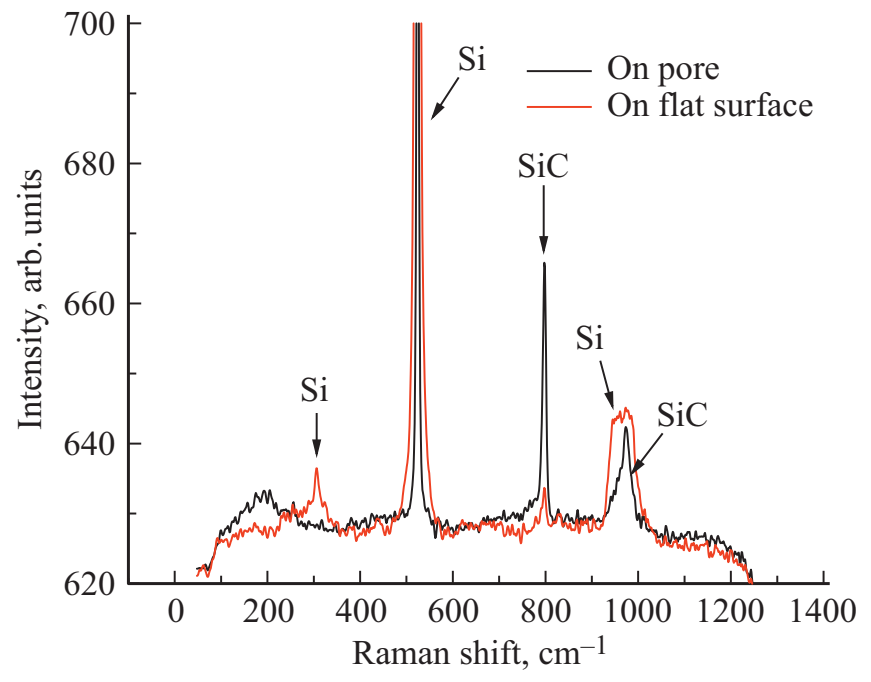

Рис. 3. Типичный рамановский спектр образцов при засветке образца над порой, и вне ее на образце $\mathrm{SiC} / \mathrm{Si}$, отожженном в течение $1 \mathrm{~min}$. Радиус пятна засветки $\sim 300 \mathrm{~nm}$.

представлены на рис. 1. В эволюции ансамбля пор были выявлены следующие закономерности. При малых временах отжига $(1-3.5 \mathrm{~min})$ под поверхностью наблюдаются отдельные дендритоподобные зародыши „пусто- ты“, не пересекающиеся друг с другом (рис. $1, a, b)$. Плотность зародышей не зависит от времени отжига и равна примерно $10^{5}-10^{6} \mathrm{~cm}^{-2}$. По мере отжига поры разрастаются и начинают сливаться друг с другом. Так, на образцах, отожженных в течение $40 \mathrm{~min}$, приповерхностная область кремния представляет собой практически сплошной пористый слой (рис. $1, d)$. При этом над пористым слоем на поверхности подложки наблюдается сплошная пленка $\mathrm{SiC}$, успевшая сформироваться даже при самом малом времени отжига (см. врезки на рис. 1). Толщина пленки $\mathrm{SiC}$, согласно эллипсометрическим измерениям и СЭМ, варьируется в диапазоне 150-180 nm для всех образцов. Пленка покрыта небольшими отверстиями (см. рис. 2) диаметром порядка 40-80 nm и плотностью, также составляющей $10^{5}-10^{6} \mathrm{~cm}^{-2}$.

По данным конфокальной рамановской микроскопии в местах расположения пор наблюдается повышенная интенсивность линии карбида кремния $\left(796 \mathrm{~cm}^{-1}\right)$ [14], что свидетельствует о том, что стенки пор состоят, либо покрыты слоем карбида кремния (см. рис. 3). Это также подтверждается и данными электронной микроскопии. Рамановские карты образцов (см. рис. 4), построенные по интенсивности линий кремния $\left(520 \mathrm{~cm}^{-1}\right)$ [15] и карбида кремния, также демонстрируют наличие отдельных небольших ( 1.5-3 $\mu \mathrm{m})$ областей с повышенной концентрацией карбида кремния при малых временах от-

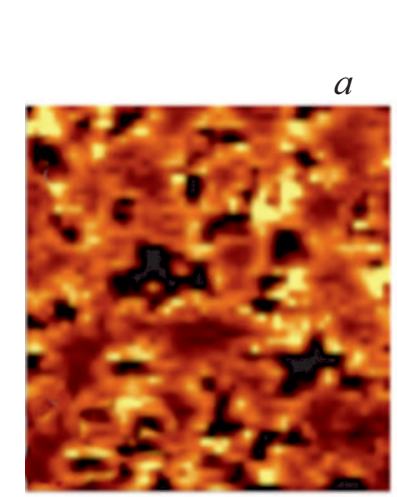

$-2 \mu \mathrm{m}$

$a$

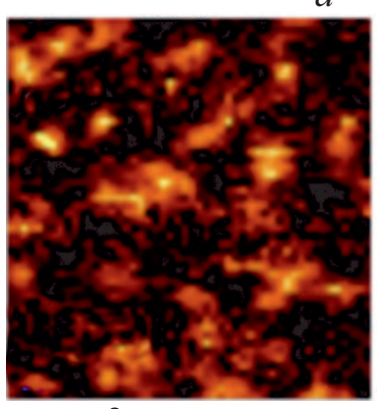

$-2 \mu \mathrm{m}$

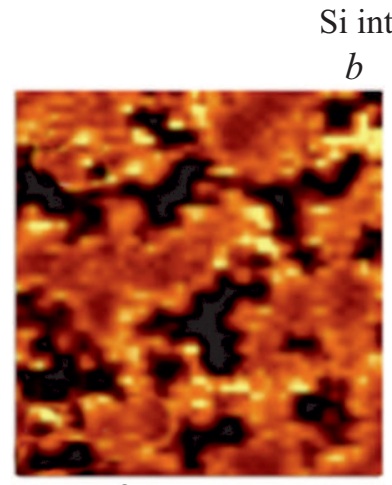

$-2 \mu \mathrm{m}$

$\mathrm{SiC}$ intensity

b

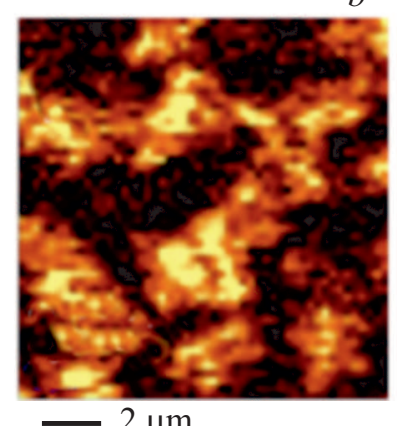

$2 \mu \mathrm{m}$

Si intensity

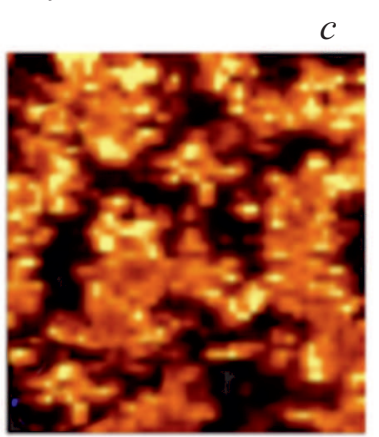

$2 \mu \mathrm{m}$

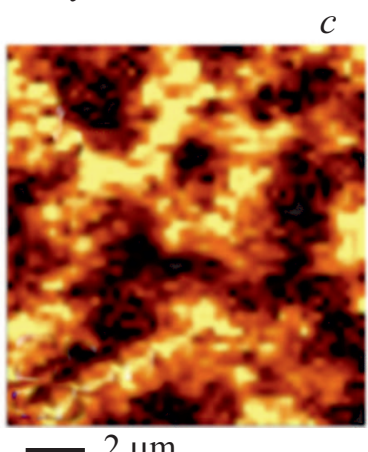

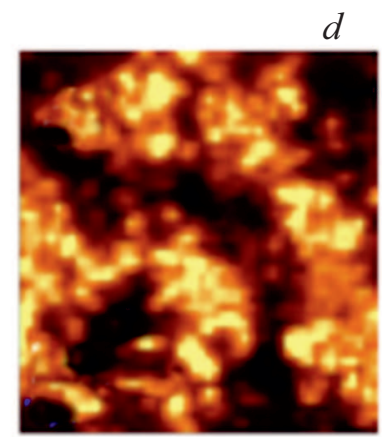

$-2 \mu \mathrm{m}$

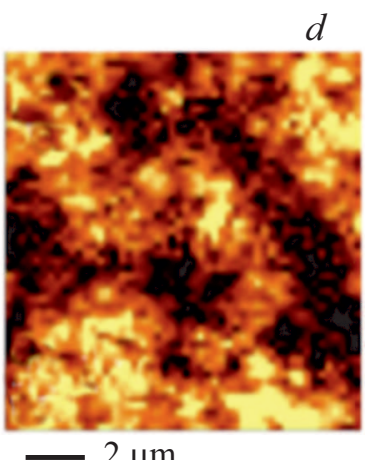

Рис. 4. Рамановские карты $(50 \times 50$ точек, $12 \times 12 \mu \mathrm{m})$ одного и того же участка поверхности каждого из образцов, построенные по интенсивности линий кремния $520 \mathrm{~cm}^{-1}$ (верхний ряд), и карбида кремния $796 \mathrm{~cm}^{-1}$ (нижний ряд) в рамановском спектре. Время отжига в атмосфере $\mathrm{CO}$ : a) $1 \mathrm{~min} ;$ b) $3.5 \mathrm{~min}$; c) $12 \mathrm{~min}$; d) $40 \mathrm{~min}$. Светлым областям соответствует повышенная интенсивность соответствующей линии. 


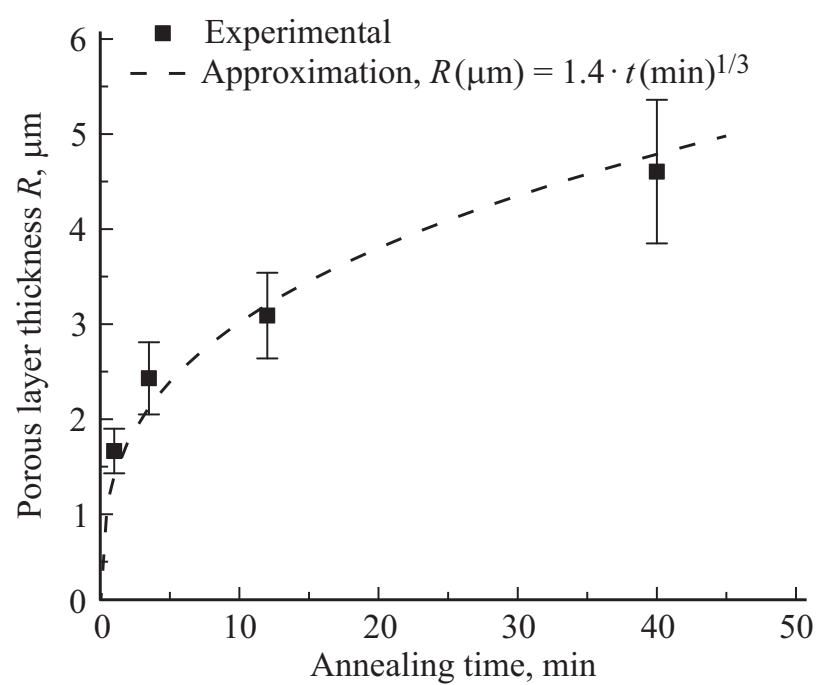

Рис. 5. Зависимость толщины сформировавшегося пористого слоя $R$ под поверхностью $\mathrm{SiC}$ от времени отжига кремния в aтмосфере CO. жига (1-3.5 min, рис. 4, $a, b)$, которые увеличиваются по мере увеличения времени отжига, и затем сливаются в непрерывные области размером порядка десятка микрон (40 min, puc. $4, d)$.

Для всех образцов была измерена средняя толщина пористого слоя (рис. 5). Для небольших времен отжига (1, 3.5, $12 \mathrm{~min})$, при которых поры еще не срослись в единый слой, эта толщина эквивалентна среднему характерному размеру $R$ (радиусу) пор (см. рис. 6). На рис. 5 нетрудно заметить, что средний радиус достаточно точно аппроксимируется степенной зависимостью от времени.

\section{3. Механизм формирования пор}

Обсуждая возможные механизмы зарождения и эволюции пор, следует упомянуть, что в работах $[8,9,16,17]$ подробно анализировался процесс проникновения газа СО вглубь кремния и формирование подобных пор,

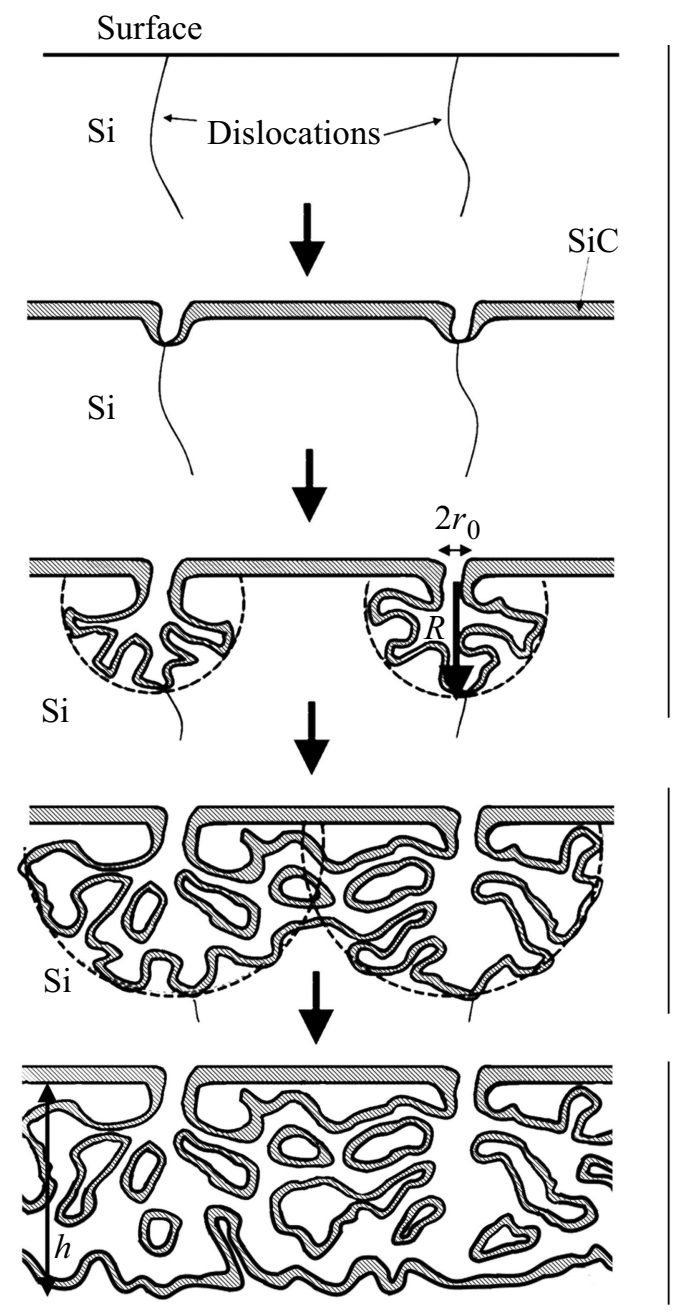

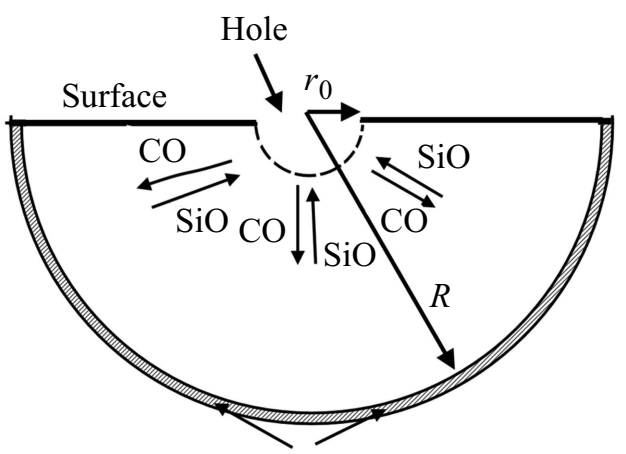

Reaction frontier

III

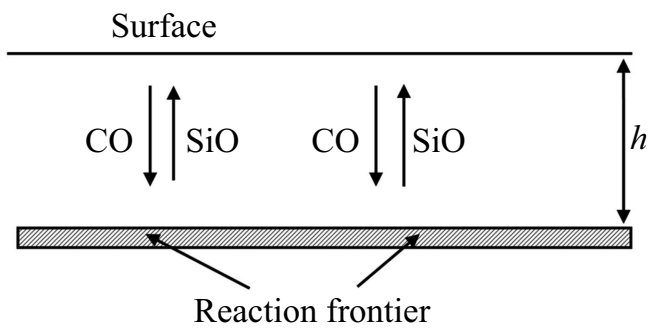

Рис. 6. Различные этапы формирования пористой структуры в приповерхностной области кремния и соответствующие им математические задачи. I - зарождение ямок травления на дислокациях и рост отдельных пор. II - срастание пор в единый пористый слой. III - равномерное увеличение толщины пористого слоя. 
однако в них рассматривался, в основном, механизм переноса массы путем диффузии [16] или дрейфа [17] $\mathrm{CO}$ и $\mathrm{SiO}$ непосредственно через кристаллическую фазу. В [17] была предсказана возможность проникновения газа СО на большие глубины (вплоть до десятка микрон) через дефекты кристаллической решетки, например дислокации или каналы. В настоящей работе развивается это предположение и предложен дислокационный механизм, который может играть существенную роль при формировании пористой структуры под поверхностью пленки. Следует также отметить, что в рамках работы мы не будем рассматривать укрупнение и разрастание пор по механизму коалесценции [18] по следующим причинам. Во-первых, общее наблюдаемое увеличение объема пор за счет химической реакции со временем значительно больше, чем объем, перераспределенный между порами, поэтому вклад коалесценции в эволюцию функции распределения пор представляется малым. Во-вторых, взаимодействие пор друг с другом происходит через объем кремния, тогда как их стенки покрыты слоем карбида кремния, и для анализа необходимо корректно учесть взаимодействие вакансионных подсистем кремния и карбида кремния, кинетику и термодинамику обмена вакансиями, что является отдельной непростой задачей, которая будет рассмотрена в одной из следующих работ. Следует также отметить, что образование пористой структуры в процессе реакции газа с твердым телом обсуждалось во многих работах $[19,20]$.

На основе полученных данных о зависимости размера и структуры пор от времени была разработана физическая модель, описывающая различные этапы роста пористого слоя, которая заключается в следующем (см. рис. 6).

1) Первым этапом является зарождение отдельных пор. Поскольку плотности пор и отверстий на поверхности пленки $\mathrm{SiC}$ - величины одного порядка, которые примерно соответствуют плотности дислокаций в исходном кремнии, целесообразно предположить, что зарождение пор происходит именно в местах выхода дислокаций на поверхность, по аналогии с хорошо изученным явлением ямок травления [21]. Также дислокационные петли, выходящие на поверхность, могут образовываться и в процессе формирования тонкой пленки $\mathrm{SiC}$, поскольку при ее появлении в приповерхностной области кремния неизбежно возникают изгибающие механические напряжения. Газ СО может эффективно проникать вглубь кремния по дислокациям и дислокационным трубкам как по диффузионному, так и, в случае большой разности давлений, по дрейфовому механизму [17]. Проникая в объем кремния, монооксид углерода преобразует его в $\mathrm{SiC}$ в области, расположенной вдоль ядра дислокации. При этом, ввиду существенной релаксации объема, ядро дислокации превращается в широкий канал, по которому может поступать дополнительный газ СО. Это приводит к дальнейшему преобразованию кремния в $\mathrm{SiC}$ и углублению поры, которая рано или поздно может натолкнуться на иные дефекты решетки, дислокации или границы зерен в объеме кремния. Подобные дефекты являются „руслом“ для дальнейшего распространения $\mathrm{CO}$ и оттока продукта реакции (1) - газообразного $\mathrm{SiO}$. В результате пора приобретает дендритообразный вид (см. врезки на рис. 1). При наличии подобных дефектов разрастание отдельного „зародыша“ поры носит изотропный характер, и форму области, охваченной дендритами, в некотором приближении, можно рассматривать как полусферу (см. рис. 6). Это хорошо видно после стравливания подложки кремния (см. рис. 4 в работе [17]). В случае, если таких дефектов нет или крайне мало, в объеме кремния могут наблюдаться отдельные „ленточные“ каналы, имеющие стенки из карбида кремния, идущие вдоль дислокаций. Наличие таких каналов также было подтверждено ранее (cм. рис. 3 в работе [17]). Другим фактором, влияющим на развитие морфологической неустойчивости в подобном канале, можно назвать и релаксацию объема при преобразовании $\mathrm{Si}$ в $\mathrm{SiC}$, при которой в случайных местах на поверхности формирующегося остова из $\mathrm{SiC}$ неизбежно появляются нанопоры, открывающие для газа СО путь к все новым областям кремния, не затронутым реакцией. Помимо этого, в поре могут наблюдаться и иные явления, например химическая реакция газов СО и $\mathrm{SiO}$ с образованием $\mathrm{SiC}$ и кислорода, в результате чего на поверхности стенок поры будет осаждаться дополнительный карбид кремния, однако этот процесс мы рассматривать в рамках настоящей работы не будем.

2) На втором этапе поры начинают постепенно срастаться и описанная выше модель уже не применима, так как приближение полусфер становится неправомерным. Момент этого перехода можно приблизительно оценить по исходной плотности пор (дислокаций): когда средний радиус пор становится равным половине расстояния между ними и, соответственно, половине расстояния между отверстиями для притока газа, происходит слияние пор.

3) На третьем этапе, когда поры сливаются в сплошной пористый слой, и расстояние между входными отверстиями для газа становится меньше толщины этого слоя, поверхность можно рассматривать как непрерывный источник $\mathrm{CO}$ и сток для $\mathrm{SiO}$. Это неизбежно приводит к необходимости решения иной задачи, а именно к рассмотрению одномерной диффузии СО от поверхности через пористый слой к области реагирования с кремнием (см. рис. 6). Похожая задача уже была решена в [16], однако там рассматривался случай диффузии газов через кристаллическую фазу.

\section{1. Эволюция пористого слоя на І-м этапе. Рост отдельных пор}

Для того чтобы описать математически основные закономерности процесса эволюции пор на первом этапе, сделаем, для простоты, следующие допущения. Во-первых, предположим, что пора, несмотря на большое количество каналов и стенок, представляет собой полую полусферу радиуса $R$, центр которой совпадает с отверстием в пленке $\mathrm{SiC}$ и которая разрастается со 
временем (см. рис. 6.). Во-вторых, предположим, что рост поры лимитируется диффузией реагентов и на ее границе $(r=R)$ соблюдается химическое равновесие, то есть $C_{\mathrm{SiO}} / C_{\mathrm{CO}}=K_{e q}$, где $C_{\mathrm{CO}}-$ концентрация монооксида углерода, $C_{\mathrm{SiO}}$ - концентрация монооксида кремния, а $K_{e q}-$ константа равновесия химической реакции (1) [16]. При этом около отверстия на поверхности радиуса $r_{0}$, через которое в пору поступает газ, поддерживается концентрация $C_{\mathrm{CO}}^{0}$, а концентрация $\mathrm{SiO}$ около этого отверстия равна нулю, ввиду быстрого обмена с атмосферой реактора. На границе полусферы $(r=R)$, в соответствии со стехиометрией реакции (1), потоки газов $\mathrm{CO}$ и $\mathrm{SiO}$ должны быть противонаправлены и по модулю равны друг другу. Тогда, в квазиравновесных условиях при малой скорости роста поры, можно записать следующую приближенную систему уравнений для распределения $\mathrm{CO}$ и $\mathrm{SiO}$ в объеме „идеальной“ поры

$$
\left\{\begin{array}{l}
\frac{d^{2} C_{\mathrm{CO}}}{d r^{2}}+\frac{2}{r} \frac{d C_{\mathrm{CO}}}{d r}=0, \\
\frac{d^{2} C_{\mathrm{SiO}}}{d r^{2}}+\frac{2}{r} \frac{d C_{\mathrm{Si}}}{d r}=0, \\
\left.C_{\mathrm{CO}}\right|_{r=r_{0}}=C_{\mathrm{CO}}^{0}, \\
\left.C_{\mathrm{SiO}}\right|_{r=r_{0}}=0, \\
\left.\frac{C_{\mathrm{SiO}}}{C_{\mathrm{CO}}}\right|_{r=R}=K_{e q}, \\
\left.D_{\mathrm{CO}} \frac{d C_{\mathrm{CO}}}{d r}\right|_{r=R}=-\left.D_{\mathrm{SiO}} \frac{d C_{\mathrm{SiO}}}{d r}\right|_{r=R},
\end{array}\right.
$$

где $D_{\mathrm{CO}}$ и $D_{\mathrm{SiO}}$ - коэффициенты диффузии.

Нетрудно показать, что решением этой системы уравнений будут следующие выражения для концентраций газов

$$
\left\{\begin{array}{l}
C_{\mathrm{CO}}(r)=\frac{C_{\mathrm{CO}}^{0}}{\left(D_{\mathrm{CO}}+D_{\mathrm{SiO}} K_{e q}\right)}\left(D_{\mathrm{CO}}+\frac{D_{\mathrm{SiO}} K_{e q} r_{0}}{\left(R-r_{0}\right)}\left(\frac{R}{r}-1\right)\right), \\
C_{\mathrm{SiO}}(r)=\frac{C_{\mathrm{CO}}^{0} D_{\mathrm{CO}} K_{e q} R}{\left(D_{\mathrm{CO}}+D_{\mathrm{SiO}} K_{e q}\right)\left(R-r_{0}\right)}\left(1-\frac{r_{0}}{r}\right) .
\end{array}\right.
$$

Зная распределение концентраций и учитывая, что $R \gg r_{0}$, найдем диффузионные потоки газов к поверхности полусферы, и тем самым, скорость увеличения радиуса рассматриваемой „идеальной“ полусферической поры

$$
\begin{aligned}
\frac{d R}{d t} & =J \omega=\left.D_{\mathrm{CO}} \frac{d C_{\mathrm{CO}}(r)}{d r} \omega\right|_{r=R} \\
& \approx \frac{C_{\mathrm{CO}}^{0} \omega}{R^{2}} \frac{D_{\mathrm{CO}} D_{\mathrm{SiO}} K_{e q} r_{0}}{\left(D_{\mathrm{CO}}+D_{\mathrm{SiO}} K_{e q}\right)},
\end{aligned}
$$

где $J$ - поток СО к поверхности поры, $\omega$ - объем пустоты, появляющийся при акте взаимодействия молекулы СО с кремнием, равный примерно $80 \AA^{3}$ [9]. Интегрируя полученное выражение, найдем зависимость среднего радиуса пор от времени

$$
R_{\text {theor }}(t) \approx \sqrt[3]{3 C_{\mathrm{CO}}^{0} \omega \frac{D_{\mathrm{CO}} D_{\mathrm{SiO}} K_{e q} r_{0}}{\left(D_{\mathrm{CO}}+D_{\mathrm{SiO}} K_{e q}\right)} t}
$$

или, подставляя типичные значения концентрации при ростовых условиях $C_{\mathrm{CO}}^{0}=\frac{P}{k T} \sim 10^{22} \mathrm{~m}^{-3}$, коэффициентов диффузии $D_{\mathrm{CO}}, D_{\mathrm{SiO}} \sim 10^{-4} \frac{\mathrm{m}^{2}}{\mathrm{~s}}$, а также константы равновесия химической реакции (1) $K_{e q} \sim 25$ [16] и среднего диаметра каналов $r_{0}$ порядка $50 \mathrm{~nm}$, получим оценку для зависимости среднего размера пор от времени

$$
R_{\text {theor }}(\mu \mathrm{m}) \approx 8.8 \sqrt[3]{t}(\min ) .
$$

Это выражение имеет полное качественное совпадение с найденным из эксперимента результатом (см. рис. 5)

$$
R_{\exp }(\mu \mathrm{m}) \approx 1.4 \sqrt[3]{t}(\min ),
$$

имеет тот же порядок и отличается от него только постоянным множителем $\sim 6.3$, который может быть обусловлен допущениями о том, что пора представляет собой идеальную полусферу без извилистых каналов и множества границ, существенно затрудняющих проникновение газа. Влияние этих каналов на распространение газа заслуживает отдельного обсуждения. Вообще говоря, при ростовых условиях длина свободного пробега молекул газа составляет порядка единиц-десятков микрон, то есть движение молекул в рассматриваемой идеальной полусфере должно было бы быть свободномолекулярным, однако пора представляет собой хаотическое переплетение множества каналов и стенок из карбида кремния (см. врезки к рис. 1). Поэтому молекула газа, перед тем как попасть в область протекания химической реакции на границе поры, испытывает большое количество диффузных переотражений и столкновений в лабиринте стенок, характерное расстояние между которыми составляет десятки-сотни нанометров. Это и обусловливает диффузионный характер распространения газа в поре и позволяет для оценки использовать диффузионный закон. Таким образом, отличительной особенностью механизма диффузии в рассматриваемой системе является то, что длина свободного пробега ограничена хаотически расположенными стенками, тогда как в классической газовой диффузии пробег ограничен столкновениями молекул друг с другом. При этом, чем больше радиус растущей поры, тем более справедливо для нее данное предположение, поскольку с ростом размера увеличивается общее количество стенок и соударений молекулы с ними.

Отметим, что пропорциональность радиуса корню кубическому из времени означает, что объем пористого слоя меняется линейно, и процесс роста поры в рамках нашей модели аналогичен надуванию воздушного шарика постоянным потоком газа. Подобная корневая зависимость наблюдается и в других системах, например при коалесценции сферических зародышей.

Второй этап эволюции пор более сложен для рассмотрения, по всей видимости, длится меньше, чем первый и третий этапы, и представляет собой их комбинацию, поэтому мы его здесь не рассматриваем. 


\section{2. Эволюция пористого слоя на III-м этапе. Равномерный рост толщины}

Зависимость толщины от времени на поздних этапах, когда поры уже срослись друг с другом и реакция (1) протекает на плоском фронте, постепенно углубляющемся в объем кристалла кремния, можно описать с помощью модифицированной модели из предыдущего раздела (см. также работу [16]). Основное отличие этого этапа от I-го заключается в том,что расстояние между центрами пор становится значительно меньше толщины пористого слоя, и на этом этапе всю поверхность можно рассматривать как непрерывный источник газа $\mathrm{CO}$ и сток газа $\mathrm{SiO}$, поэтому задача представляет собой диффузию в полуплоскость $y>0$ (см. рис. 6), где ось $y$ направлена вглубь кристалла. Граничные условия остаются теми же. На поверхности поддерживается постоянная концентрация монооксида углерода $C_{\mathrm{CO}}^{0}$ и нулевая концентрация газа $\mathrm{SiO}$. У фронта реакции на глубине $h$ (она же - толщина пористого слоя) поддерживается химическое равновесие, и соотношение концентраций газов определяется константой химической реакции (1) $C_{\mathrm{SiO}} / C_{\mathrm{CO}}=K_{e q}$. Потоки газов $\mathrm{CO}$ и $\mathrm{SiO}$ у фронта реакции противонаправлены и равны по модулю. Система уравнений, описывающая распределение концентраций в рамках этой модели, выглядит так

$$
\left\{\begin{array}{l}
\frac{d^{2} C_{\mathrm{CO}}}{d y^{2}}=0, \\
\frac{d^{2} C_{\mathrm{SiO}}}{d y^{2}}=0, \\
\left.C_{\mathrm{CO}}\right|_{y=0}=C_{\mathrm{CO}}^{0}, \\
\left.C_{\mathrm{SiO}}\right|_{y=0}=0, \\
\left.\frac{C_{\mathrm{SiO}}}{C_{\mathrm{CO}}}\right|_{y=h}=K_{e q}, \\
\left.D_{\mathrm{CO}} \frac{d C_{\mathrm{CO}}}{d y}\right|_{y=h}=-\left.D_{\mathrm{SiO}} \frac{d C_{\mathrm{SiO}}}{d y}\right|_{y=h},
\end{array}\right.
$$

где $y$ - расстояние от поверхности. Решением этой системы, как нетрудно показать, являются функции

$$
\begin{gathered}
C_{\mathrm{CO}}(y)=\frac{C_{\mathrm{CO}}^{0}}{\left(D_{\mathrm{CO}}+D_{\mathrm{SiO}} K_{e q}\right)}\left(D_{\mathrm{CO}}+\frac{D_{\mathrm{SiO}} K_{e q}(h-y)}{h}\right) \\
C_{\mathrm{SiO}}(y)=\frac{C_{\mathrm{CO}}^{0} D_{\mathrm{CO}} K_{e q} y}{\left(D_{\mathrm{CO}}+D_{\mathrm{SiO}} K_{e q}\right) h}
\end{gathered}
$$

По аналогии с предыдущим разделом, найдем поток газа $\mathrm{CO}$ к плоскому фронту реакции и скорость перемещения фронта

$$
\begin{aligned}
\frac{d h}{d t} & =J \omega=\left.D_{\mathrm{CO}} \frac{d C_{\mathrm{CO}}(r)}{d y} \omega\right|_{y=h} \\
& =\frac{C_{\mathrm{CO}}^{0} \omega}{h} \frac{D_{\mathrm{CO}} D_{\mathrm{SiO}} K_{e q}}{\left(D_{\mathrm{CO}}+D_{\mathrm{SiO}} K_{e q}\right)} .
\end{aligned}
$$

Интегрируя полученное выражение, получим выражение для зависимости толщины пористого слоя $h$ от времени

$$
h(t)=\sqrt{\frac{\left(2 C_{\mathrm{CO}}^{0} \omega D_{\mathrm{CO}} D_{\mathrm{SiO}} K_{e q} t\right.}{\left(D_{\mathrm{CO}}+D_{\mathrm{SiO}} K_{e q}\right)}}+h_{0},
$$

где толщина $h_{0}$ примерно равна максимальному радиусу пор на предыдущем этапе роста, или, как было показано ранее, половине среднего расстояния между точками зарождения пор (точками выхода дислокаций на поверхность).

\section{4. Заключение}

Рассмотрен процесс порообразования при взаимодействии газа СО с кристаллическим кремнием, в результате которого последний преобразуется в карбид кремния. Продемонстрированы характерные черты процесса: зарождение одиночных непересекающихся пор под поверхностью кремния, их укрупнение, развитие дендритоподобной структуры их стенок и срастание в единый пористый слой. Изучена временна́я эволюция средней толщины пористого слоя при выдержке образца в атмосфере СО и показано, что толщина на начальных этапах пропорциональна кубическому корню из времени. Предложена модель, описывающая процесс формирования пористого слоя как на начальных этапах, когда слой представляет собой обособленные поры, так и на поздних, когда поры срастаются и движутся вглубь единым плоским фронтом. Теоретически показано, что в последнем случае толщина пористого слоя пропорциональна квадратному корню из времени. Модель, несмотря на свою простоту, позволяет качественно оценить влияние основных параметров ростового процесса на эволюцию пористого слоя в приповерхностной области $\mathrm{SiC} / \mathrm{Si}$ и дать оценку его толщины при заданных условиях роста: давлении $\mathrm{CO}$, времени отжига и температуры, которой определяются коэффициенты диффузии и константа равновесия химической реакции кремния с СО. Модель может быть использована для подбора режима формирования пористого слоя заданной толщины без проведения большого количества экспериментов.

Электронно-микроскопические исследования выполнены с использованием оборудования федерального ЦКП „Материаловедение и диагностика в передовых технологиях“, поддержанного Министерством образования и науки России (Уникальный идентификатор проекта RFMEFI62117X0018), а также с использованием уникальной научной установки „Физика, химия, и механика кристаллов и тонких пленок“ (ИПМаш РАН, СанктПетербург).

\section{Список литературы}

[1] R.S. Pengelly, S.M. Wood, J.W. Milligan, S.T. Sheppard, W.L. Pribble. IEEE Trans. Microwave Theory Tech. 60, 1764 (2012).

[2] R. Liu, F.A. Ponce, A. Dadgar, A. Krost. Appl. Phys. Lett. 83, 860 (2003). 
[3] F.A. Ponce, D.P. Bour. Nature 386, 351(1997).

[4] S.N. Mohammad, H. Morkoç. Progr. Quantum Electron., 20, 361 (1996).

[5] S. Nakamura. Jpn. J. Appl. Phys. 30, L1705 (1991).

[6] K. Linthicum, T. Gehrke, D. Thomson, K. Tracy, E. Carlson, T. Smith, T. Zheleva, C. Zorman, M. Mehregany, R. Davis. MRS Internet J. Nitride Semicond. Res. 4S1 G4.9 (1999).

[7] S. Guha, N.A. Bojarczuk. Appl. Phys. Lett. 72, 415 (1998).

[8] S.A. Kukushkin, A.V. Osipov. J. Phys. D. 47, 313001 (2014).

[9] S.A. Kukushkin, A.V. Osipov. J. Appl. Phys. 113, 024909 (2013).

[10] S.L. Khrypko, V.V. Kidalov. J. Nano- Electron. Phys. 8, 04071 (2016).

[11] V.V. Kidalov, S.A. Kukushkin, A.V. Osipov, A. V. Redkov, A.S. Grashchenko, I.P. Soshnikov, M.E. Boiko, M.D. Sharkov, A.F. Dyadenchuk. Mater. Phys. Mech. 36, 39 (2018).

[12] V.N. Bessolov, D.V. Karpov, E.V. Konenkova, A.A. Lipovskii, A.V. Osipov, A.V. Redkov, I.P. Soshnikov, S.A. Kukushkin. Thin Solid Films 606, 74 (2016).

[13] S.A. Kukushkin, S.S. Sharofidinov, A.V. Osipov, A.V. Redkov, V.V. Kidalov, A.S. Grashchenko, I.P. Soshnikov, A.F. Dydenchuk. ECS J. Solid State Sci. Technol. 7, 480 (2018).

[14] S.I. Nakashima, H. Harima. Phys. Status Solidi A 162, 39 (1997).

[15] H. Richter, Z.P. Wang, L. Ley. Solid State Commun. 39, 625 (1981).

[16] С.А. Кукушкин, А.В. Осипов. ФТТ 50, 1188 (2008).

[17] S.A. Kukushkin, A.V. Osipov. Physica B: Condens. Matter 512, 26 (2017).

[18] В.В. Слезов, В.Б. Шикин. ФТТ 6, 7(1964).

[19] M. Sahimi, T.T. Tsotsis. Chem. Eng. Sci. 43, 113 (1988).

[20] P. Sun, J.R. Grace, C.J. Lim, E.J. Anthony. Chem. Eng. Sci. 63, 57 (2008).

[21] K. Sangwal. Etching of crystals: theory, experiment and application. Elsevier., Amsterdam (2012).

Редактор Ю.Э. Китаев 